Materiales de Construcción Vol. 58, 289-290, 81-95 enero-junio 2008 ISSN: $0465-2746$ eISSN: $1988-3226$

\title{
La composición mineralógica de la arenisca y su influencia en la deposición de dióxido de azufre
}

\author{
The mineralogical composition of sandstone and its \\ effect on sulphur dioxide deposition
}

\author{
$\underline{\text { U. Müller }}{ }^{(*)}$
}

Recepción/Received: 23-V-07

Aceptación/Accepted: 11-X-07

Publicación online/Online publishing: 14-II-08

\section{RESUMEN}

Los contaminantes atmosféricos son con frecuencia responsables de la aceleración de la degradación de la piedra en los edificios y monumentos históricos de las zonas urbanas. Son muchos los factores que influyen de manera decisiva en el comportamiento de las piedras naturales ante los contaminantes gaseosos. Dos de esos factores son la composición de la atmósfera y el tipo de piedra. Las areniscas en particular son propensas al ataque de los contaminantes atmosféricos debido a su naturaleza porosa, pero muchas de las reacciones de las areniscas no carbonatadas con estos gases no se conocen bien. El estudio presentado tiene por objeto comprender los procesos y factores que controlan el distinto comportamiento de las areniscas respecto al dióxido de azufre. Para el estudio se investigaron siete areniscas distintas procedentes del sur y del este de Alemania. Las areniscas mostraban una diferencia significativa en la composición de su aglomerado. Por lo que respecta a la absorción de $\mathrm{SO}_{2}$, las distintas variedades de arenisca mostraron un comportamiento completamente diferente. Curiosamente, la cantidad de $\mathrm{SO}_{2}$ depositada no estaba relacionada con la superficie específica de las areniscas, este parámetro está relacionado con el contenido en óxidos de hierro.

Palabras clave: arenisca, contaminantes, deposición, deterioro, catalizador.

\section{SUMMARY}

Air pollutants often accelerate stone deterioration in historical buildings and monuments in urban areas. Many factors condition natural stone behaviour with respect to gaseous pollutants. Two of the more prominent of such factors are the composition of the atmosphere and the type of stone. Due to their porosity, sandstones are particularly vulnerable to air pollutant attack. Many of the reactions between noncarbonaceous sandstones and these gases are not well understood, however. The present study aimed to acquire an understanding of the processes and factors governing sandstone behaviour when exposed to sulphur dioxide. Seven different sandstones from southern and eastern Germany were analyzed for the study. The binder composition of the stones varied significantly. They also exhibited completely different behaviour in connection with $\mathrm{SO}_{2}$ deposition. Interestingly, while the amount of $\mathrm{SO}_{2}$ deposited was unrelated to the specific surface area of the sandstones, this parameter was closely correlated to the iron oxide content.

Keywords: sandstone, pollutants, deposition, deterioration, catalyst.

(*) Federal Institute for materials research and testing (BAM) (Berlín, Alemania) 


\section{INTRODUCCIÓN}

La arenisca fue y sigue siendo un material muy utilizado en los sillares, elementos decorativos y revestimiento de edificios y monumentos. Su comportamiento como material de construcción depende en gran medida de sus propiedades intrínsecas, tales como su composición, su resistencia, su porosidad, etc. En el caso de la arenisca, estas propiedades intrínsecas pueden ser muy diferentes debido a que la arenisca es un material compuesto por varios elementos: granos detríticos de tamaño variable, una fina matriz cementante y un sistema poroso abierto. En ocasiones, la matriz cementante, que suele estar formada por minerales arcillosos y óxidos, puede tener un contenido muy bajo de éstos. En este caso se consigue la cohesión mediante granos que se unieron durante la diagénesis. Otro componente de la matriz puede ser el cemento carbonatado formado durante la sedimentación o la diagénesis. A menudo, los clastos de la arenisca están ligados entre sí por los tres mecanismos de unión a la vez (matriz arcillosa, sobrecrecimiento de granos y cemento carbonatado) (1).

Los edificios que constan de elementos de arenisca reaccionan de forma distinta ante el clima y las tensiones ambientales (p. ej., temperatura, lluvia, heladas, viento). Algunas variedades de arenisca son más propensas que otras al deterioro y muestran daños importantes después de poco tiempo. En concreto, las areniscas arcillosas y carbonáticas parecen ser más vulnerables ante las influencias ambientales que las areniscas con una elevada proporción de sobrecrecimiento de los granos y un bajo contenido de arcilla y carbonato (2-4). Pero no sólo influye en la durabilidad de la arenisca la composición del aglomerado. Otro factor igualmente importante es la porosidad de la arenisca, incluidos el número y el tamaño de los poros y su conectividad. El sistema poroso influye en el transporte y distribución de los iones disueltos en el agua o de las sustancias gaseosas por el interior de la piedra (5), pero además su papel es fundamental en el grado de daño que estas sustancias puedan causar al cristalizar.

En un entorno con contaminantes atmosféricos, los efectos que provocan el deterioro de la piedra pueden aumentar y acelerarse de forma considerable $(6,7)$. En concreto, una porosidad abierta, como la de la arenisca, permite que los contaminantes gaseosos (p. ej., $\mathrm{SO}_{2}, \mathrm{NOx}, \mathrm{NH}_{3}$, etc.) se introduzcan en el material e interactúen con sus componentes. Las reacciones químicas con los gases atmosféricos nocivos no suelen producirse directamente, sino que incluyen complejos mecanismos de procesos de absorción en superficie seguidos de reacciones con componentes del aglomerado o de los clastos. Como se ha demostrado en numerosos estudios, la piedra calcárea tiene una fuerte reacción con los contaminantes gaseosos, en parte por la

\section{INTRODUCTION}

Sandstone has been and continues to be widely used in building and monument structures, decoration and cladding. Its performance as a building material is highly dependent upon intrinsic properties such as composition, strength and porosity. Such properties may vary widely in sandstone, a material with many components and a detrital structure with different sized grains, a fine binder matrix and an open pore network. The binder matrix, usually consisting of clay minerals and oxides, sometimes accounts for only a small proportion of the stone, in which case cohesion is provided by grains that grew together during diagenesis. Carbonate cement, an additional binder component formed during sedimentation or diagenesis, may also be present. The grain components in sandstone are often bound together by all three mechanisms (clayey matrix, grain overgrowth, carbonate cement), whose respective proportions may vary (1).

Buildings consisting in or containing sandstone elements react differently to the prevailing climate and environmental stress induced by temperature, rain, frost or wind. Some varieties of sandstone are more prone to deterioration than others, exhibiting considerable damage after short periods of time. Clayey and carbonaceous sandstones seem to be more vulnerable to environmental agents than sandstones with a high proportion of grain overgrowth and a low clay and carbonate content (2-4). But binder composition is not the only characteristic that determines sandstone durability. Equally important is stone porosity, including pore number, size and connectivity. The pore network not only affects the transport and distribution of dissolved ions or gaseous substances inside the stone (5), but also plays a prominent role in the extent of damage caused by the crystallization of these substances.

Actions that lead to stone deterioration may be intensified considerably in polluted environments $(6,7)$. In particular, open pore networks such as in sandstone allow gaseous pollutants $\left(\mathrm{SO}_{2}, \mathrm{NOx}, \mathrm{NH}_{3}\right.$, etc. ) to penetrate the material and interact with its components. The chemical reactions induced by harmful atmospheric gases do not usually take place directly, but rather involve complex surface sorption processes followed by interaction with binder constituents or grain components. Many studies have shown that calcareous stone reacts intensely with gaseous pollutants partly via carbonate dissolution and partly via sulphate or nitrate crystallization on the surface or in the pore network (8-12). Dannecker and Steiger 
disolución de carbonato pero también por la cristalización de los sulfatos o nitratos sobre la superficie o dentro del sistema poroso (8-12). Dannecker y Steiger (13) afirmaron incluso que la principal fuente de sales en las fachadas de piedra eran la deposición de dióxido de azufre y su reacción con el sustrato pétreo.

Aunque se conoce bastante bien la deposición del dióxido de azufre y de otros gases atmosféricos sobre la roca calcárea, sigue existiendo cierto desconocimiento respecto a los mecanismos de deposición de estos contaminantes en piedras no calcáreas, como algunas areniscas. Wittenburg y otros (14) compararon la velocidad media de deposición en distintas areniscas expuestas a diferentes condiciones ambientales. A pesar de que las concentraciones de gas no fueron constantes, era evidente que la velocidad de deposición estaba claramente influida por el tipo de arenisca, es decir, por la naturaleza del componente del aglomerado. La deposición de contaminantes gaseosos dependen de muchos factores. Aparte del tipo y de la concentración del componente gaseoso en el aire, la composición total del gas también cumple una función importante. Ha quedado demostrado que las velocidades de degradación producidas por mezclas de gases eran muy superiores a las producidas por un solo tipo de gas $(15,16)$. Las mayores velocidades de degradación sobre un mismo tipo de piedra y en las mismas condiciones se producían con una mezcla de $\mathrm{NO}_{2}, \mathrm{SO}_{2}, \mathrm{O}_{3}$ y $\mathrm{H}_{2} \mathrm{O}$. Al compararlas con esta mezcla, se vio que las velocidades de degradación de $\mathrm{SO}_{2}$ y NO $\mathrm{N}_{2}$ por separado eran inferiores en una orden de magnitud (16). Aparte de la composición y del tipo de los contaminantes gaseosos presentes en la atmósfera, también podría influir en la deposición de gases la propia piedra. Haneef y otros $(15,16)$ indicaron que es posible que los óxidos metálicos finamente dispersados en la piedra puedan actuar como catalizador en la oxidación de $\mathrm{SO}_{2}$ a $\mathrm{SO}_{3}$ en una solución acuosa. Sin embargo, otros estudios apuntan en la dirección contraria. Böke y otros (17) apenas observaron actividad catalítica en los óxidos metálicos. Dichos autores investigaron la deposición de $\mathrm{SO}_{2}$ en polvo de mármol mezclado con distintas sustancias. Se tratará esta cuestión más adelante, al comentar los resultados del presente trabajo.

Basándose en estas conclusiones, a veces contradictorias, se pretendía investigar la influencia de la composición de la piedra en la deposición y fijación del dióxido de azufre en la arenisca. El estudio se centró principalmente en la composición del aglomerado y en su función en la fijación de $\mathrm{SO}_{2}$ en la matriz de la piedra, aunque también se consideraron parámetros físicos como la superficie específica y la porosidad. Se eligieron areniscas con un aglomerado arcilloso y sobrecrecimiento de granos representativas en gran medida del espectro de areniscas utilizadas como material de construcción. También se eligió una variedad con cemento carbonatado para poder
(13) went so far as to assert that the sulphur dioxide deposition and its reaction with the stone substrate is the main source of salts in stone facades.

Whereas the deposition of sulphur dioxide and other atmospheric gases on calcareous stone is fairly well understood, knowledge is still lacking about the deposition mechanisms of these pollutants on noncalcareous stones such as sandstone. Wittenburg et al. (14) compared the average deposition velocity of different sandstones exposed to a number of environmental conditions. Even though gas concentration was not constant, the deposition velocity was clearly influenced by the type of sandstone, or more precisely, the type of binder component. The deposition of gaseous pollutants depends on many factors. In addition to the type of a simple gas and its concentration in the air, gas composition, the number of gas spezies occuring together also plays an important role. Degradation generated by mixed gases has been shown to take place much more swiftly than when induced by a single gas $(15,16)$. For any given stone and test conditions, the highest degradation rates were found for a mix of $\mathrm{NO}_{2}, \mathrm{SO}_{2}, \mathrm{O}_{3}$ and $\mathrm{H}_{2} \mathrm{O}$. The degradation rates generated by $\mathrm{SO}_{2}$ and $\mathrm{NO}_{2}$ individually were a full magnitude lower (16). Moreover, stone characteristics may also affect gas deposition. Haneef et al. $(15,16)$ suggested that finely dispersed metal oxides in the stone may catalyze the oxidation of $\mathrm{SO}_{2}$ to $\mathrm{SO}_{3}$ in aqueous solutions. Other studies yielded results suggesting exactly the opposite, however Böke et al. (17) found almost no catalytic activity in metal oxides when analyzing $\mathrm{SO}_{2}$ deposition in marble powder mixed with different substances. Their results will be discussed below in conjunction with the findings of the present study.

Given these somewhat contradictory reports, the aim of this research was to study the effect of stone composition on sulphur dioxide deposition and fixation in sandstone. While the study focused primarily on binder composition and its role in $\mathrm{SO}_{2}$ fixation in the stone matrix, physical parameters such as specific surface area and porosity were also analyzed. Most of the sandstone types chosen had a clayey binder and grain overgrowth, providing a good cross section of sandstone used in buildings. One variety with a carbonate cement was also chosen to 
comparar la magnitud de los efectos entre la arenisca no carbonatada y la arenisca carbonatada.

\section{CARACTERÍSTICAS DE LA ARENISCA ESTUDIADA}

Las areniscas estudiadas constaban de arenitas procedentes de distintas canteras del sur y el este de Alemania (Baden-Wurtemberg y Sajonia, respectivamente), utilizadas en la construcción de numerosos edificios históricos. En la Tabla 1 se indica la procedencia geográfica y geológica de los materiales empleados; en la Tabla 2, la composición de su aglomerado y, en la Tabla 3 sus propiedades físicas y mecánicas. Los clastos constaban en su mayoría de cuarzo y fragmentos de roca. Tan sólo las areniscas de Maulbronn y Pfinztal tenían un contenido de feldespato superior, de entre el 20 y el $25 \%$ de su volumen. Todas las areniscas contenían un aglomerado arcilloso, be able to compare the magnitude of the effects in noncarbonaceous and carbonaceous sandstone.

\section{SANDSTONE CHARACTERISTICS}

The sandstone studied included arenites from quarries in southern (Baden-Wuertemberg) and eastern Germany (Saxony) that are found in many historical buildings. Table 1 lists the origin and geologic time, Table 2 the binder composition and Table 3 the physical and mechanical properties of the materials. The grain components consisted primarily in rock fragments and quartz. Only the Maulbronner and Pfinztaler stones had a high feldspar content, ranging from 20 to $25 \%$ by volume. Clayey binder was present in all the sandstones, although the Reinhardsdorfer and Postaer stones had a very low clay mineral content. Cohesion in these two sandstones was

Tabla 1 / Table 1

Procedencia geográfica y geológica y abreviatura de las areniscas estudiadas.

Origin, geologic time and labeling of the sandstones studied.

\begin{tabular}{|l|l|l|c|}
\hline Arenisca / Sandstone & Procedencia / Origin & Periodo / Age & Abreviatura / Label \\
\hline Posta / Postaer & Wehlen, Sajonia / Wehlen, Saxony & Cretáceo / Cretaceous & Po \\
\hline $\begin{array}{l}\text { Reinhardtsdorf / } \\
\text { Reinhardtsdorfer }\end{array}$ & Reinhardtsdorf, Sajonia / Reinhardsdorf, Saxony & Cretáceo / Cretaceous & $\mathrm{R}$ \\
\hline Cotta / Cottaer & $\begin{array}{l}\text { Cotta, sudeste de Pirna, Sajonia / Cotta, south east of } \\
\text { Pirna, Saxony }\end{array}$ & Cretáceo / Cretaceous & $\mathrm{C}$ \\
\hline Donzdorf / Donzdorfer & $\begin{array}{l}\text { Donzdorf, Baden-Wurtemberg / Donzdorf, Baden- } \\
\text { Wuerttemberg }\end{array}$ & Jurásico / Jurassic & $\mathrm{D}$ \\
\hline Maulbronn / Maulbronner & $\begin{array}{l}\text { Maulbronn, Baden-Wurtemberg / Maulbronn, Baden- } \\
\text { Wuerttemberg }\end{array}$ & Triásico / Triassic & $\mathrm{M}$ \\
\hline Pliezhaus / Pliezhauser & $\begin{array}{l}\text { Rübgarten, cerca de Tubinga, Baden-Wurtemberg / } \\
\text { Rübgarten near Tübingen, Baden-Wuerttemberg }\end{array}$ & Triásico / Triassic & $\mathrm{PI}$ \\
\hline Pfinztal / Pfinztaler & $\begin{array}{l}\text { Grünwettersbach, Baden-Wurtemberg / } \\
\text { Grünwettersbach, Baden-Wuerttemberg }\end{array}$ & Triásico / Triassic & $\mathrm{Pf}$ \\
\hline
\end{tabular}

aunque en el caso de las areniscas de Reinhardtsdorf y Posta su contenido de minerales arcillosos era muy bajo. Estas dos areniscas estaban cohesionadas principalmente mediante el sobrecrecimiento durante la diagénesis de los granos de cuarzo y feldespato. Sólo la arenisca de Pliezhaus contenía carbonato (contenido de $\mathrm{CO}_{2}=0,93 \%$ de la masa) en forma de cemento de esparita dolomítica y calcítica. La composición de la fracción arcilla se determinó mediante difracción por rayos $X(X R D)$ según el procedimiento descrito en (18). Con la excepción de la arenisca de Pfinztal, cuyo único mineral arcilloso era la ilita, todas las demás contenían varias fases de minerales arcillosos características de cada una de las areniscas. Normalmente constaban de caolinita, ilita y una fase mixta (Tabla 2). primarily the result of the diagenetic overgrowth of quartz and feldspar grains. Only the Pliezhauser sandstone contained carbonates ( $\mathrm{CO}_{2}$ content $=0.93 \%$ by mass) in the form of dolomitic and calcitic sparite cement. The composition of the clay fraction was determined by $X$-ray diffraction (XRD) as described in (18). Except for the Pfinztaler sandstone, in which illite was the only clay mineral, all others contained a number of such phases, in proportions distinctly characteristic of each sandstone. The phases most commonly found were kaolinite, illite and a mixed layer (Table 2). 
Tabla 2 / Table 2

Composición del aglomerado y contenido total de hierro. Binder composition and total iron content.

\begin{tabular}{|c|c|c|c|c|}
\hline $\begin{array}{l}\text { Arenisca } \\
\text { Sandstone }\end{array}$ & $\begin{array}{l}\text { Minerales arcillosos / } \\
\text { Clay minerals }\end{array}$ & $\begin{array}{c}\text { Otros } \\
\text { componentes de } \\
\text { la fase de union } \\
\text { / Other binder } \\
\text { components }\end{array}$ & $\begin{array}{l}\text { Sobrecrecimiento } \\
\text { de granos de / } \\
\text { Composition of } \\
\text { grain overgrowth }\end{array}$ & $\begin{array}{c}\text { Contenido de } \\
\mathrm{Fe}_{2} \mathrm{O}_{3 \text { tot }}(\% \text { de la } \\
\text { masa) } / \mathrm{Fe}_{2} \mathrm{O}_{3 t o t} \\
\text { content } \\
\text { (\% by mass) }\end{array}$ \\
\hline Cotta / Cottaer & $\begin{array}{l}\text { caolinita. capa mixta (CM) de ilita y } \\
\text { esmectita. ilita (glauconita) / kaolinite. } \\
\text { illite-smectite mixed layer (ML). illite } \\
\text { (glaukonite) }\end{array}$ & - & - & 0.27 \\
\hline $\begin{array}{l}\text { Maulbronn / } \\
\text { Maulbronner }\end{array}$ & $\begin{array}{l}\text { CM de clorita y vermiculita. ilita / } \\
\text { chlorite-vermiculite ML.. illite }\end{array}$ & $\begin{array}{l}\text { hematites / } \\
\text { hematite }\end{array}$ & $\begin{array}{l}\text { feldespato potásico. } \\
\text { cuarzo / potassium } \\
\text { feldspar. quartz }\end{array}$ & 4.47 \\
\hline $\begin{array}{l}\text { Reinhardsd. / } \\
\text { Reinhardtsd. }\end{array}$ & $\begin{array}{l}\text { caolinita. CM de ilita y esmectita. ilita } \\
\text { / kaolinite. illite-smectite ML. illite }\end{array}$ & $\begin{array}{l}\text { lepidocrocita / } \\
\text { lepidocrocite }\end{array}$ & cuarzo / quartz & 0.39 \\
\hline Posta / Postaer & $\begin{array}{l}\text { caolinita. ilita. CM de clorita y } \\
\text { esmectita / kaolinite. illite. chlorite- } \\
\text { smectite ML }\end{array}$ & - & cuarzo / quartz & 0.13 \\
\hline $\begin{array}{l}\text { Pliezhaus / } \\
\text { Pliezhauser }\end{array}$ & $\begin{array}{l}\text { caolinita. ilita. CM de ilita y esmectita. } \\
\text { CM de clorita y esmectita / kaolinite. } \\
\text { illite. illite-smectite ML. chlorite- } \\
\text { smectite ML }\end{array}$ & $\begin{array}{l}\text { dolomía. calcita / } \\
\text { dolomite. calcite }\end{array}$ & cuarzo / quartz & 0.22 \\
\hline $\begin{array}{l}\text { Pfinztal / } \\
\text { Pfinztaler }\end{array}$ & ilita / illite & $\begin{array}{l}\text { hematites / } \\
\text { hematite }\end{array}$ & $\begin{array}{l}\text { feldespato potásico. } \\
\text { cuarzo / potassium } \\
\text { feldspar. quartz }\end{array}$ & 2.10 \\
\hline $\begin{array}{l}\text { Donzdorf / } \\
\text { Donzdorfer }\end{array}$ & $\begin{array}{l}\text { ilita. caolinita. CM de ilita y esmectita } \\
\text { / illite. kaolinite. illite-smectite } M L\end{array}$ & goetita / goethite & cuarzo / quartz & 1.62 \\
\hline
\end{tabular}

Cuatro de las areniscas analizadas contenían además óxido de hierro o hidróxido de hierro en la fracción arcilla (Tabla 2). La concentración total de hierro (determinada mediante análisis de fluorescencia de rayos $X$ de longitud de onda dispersiva) presentaba unos valores variables, como puede verse en la Tabla 2 . En un análisis modal de la arenisca mediante microscopía de luz polarizada no se halló un contenido importante de máfica, una roca que contiene hierro, por lo que se supuso que la mayoría del hierro se encontraba en el cemento en forma de fases de óxido o hidróxido. Aunque no se cuantificó específicamente el contenido de matriz cementante de las areniscas, el procedimiento de separación de los minerales arcillosos para la XRD permitió establecer la siguiente orden ascendente: Posta < Pliezhaus < Reinhardtsdorf < Donzdorf < Pfinztal $<$ Cotta $<$ Maulbronn.

Las propiedades físicas y mecánicas de los distintos tipos de arenisca eran tan variadas como la composición de sus aglomerados. En la Tabla 3 se resumen los principales parámetros. La porosidad total varió entre el $11 \%$ y el $29 \%$, mientras que la resistencia a la compresión uniaxial dio unos resultados de entre 20 y $122 \mathrm{MPa}$. De la superficie específica se obtuvo un valor muy bajo en el caso de la arenisca de Posta $\left(0,06 \mathrm{~m}^{2} / \mathrm{g}\right)$ y valores bastante elevados para las areniscas de Cotta y Maulbronn (entre 5 y
Four of the sandstones studied also contained iron oxide or iron hydroxide in the clay fraction (Table 2). The total iron concentration (determined by wave length dispersive $X$-ray fluorescence analysis) varied, as shown in Table 2. A polarized light microscope modal analysis of the sandstone revealed no major detrital mafic, or iron containing minerals. It was therefore assumed that most of the iron was found in the binder fraction in the form of oxides or hydroxides. Although the binder matrix content in the sandstones was not specifically quantified, the procedure for separating the clay minerals for XRD revealed the following estimate (in ascending order): Postaer < Pliezhauser < Reinhardsdorfer < Donzdorfer < Pfinztaler $<$ Cottaer < Maulbronner.

The more diverse the binder composition, the more variable were sandstone physical and mechanical properties. The main parameters are summarized in Table 3. Total porosity ranged from 11 to $29 \%$ and the uniaxial compressive strength values from 20 to $122 \mathrm{MPa}$. The specific surface area was very low $\left(0.06 \mathrm{~m}^{2} / \mathrm{g}\right)$ in the Postaer sandstone and fairly high in Cottaer and Maulbronner sandstone (5 - $6.5 \mathrm{~m}^{2} / \mathrm{g}$ ). Although the lowest porosity (and highest strength) and smallest pore sizes observed 
$\left.6,5 \mathrm{~m}^{2} / \mathrm{g}\right)$. Aunque la arenisca de Pfinztal tenía la porosidad más baja (y el mejor comportamiento mecánico) y el tamaño de poros más pequeño de todos los materiales estudiados, también tenía una superficie específica mediana (3 $\mathrm{m}^{2} / \mathrm{g}$ ) debido al mayor contenido de matriz cementante. in the study were found in Pfinztaler sandstone, this material had a moderately large surface area of $3 \mathrm{~m}^{2} / \mathrm{g}$, due to its higher binder matrix content.

Tabla 3 / Table 3

Composición del aglomerado y contenido total de hierro. Binder composition and total iron content.

\begin{tabular}{|c|c|c|c|c|c|c|}
\hline & $\begin{array}{c}A_{b} \\
\% \text { de masa / } \\
\text { by mass }\end{array}$ & $\underset{g /\left(m^{2} s^{0.5}\right)}{C}$ & $\begin{array}{c}\rho_{b} \\
\mathbf{k g} / \mathbf{m}^{3}\end{array}$ & $\begin{array}{c}\mathbf{p} \\
\%\end{array}$ & $\begin{array}{c}\mathbf{R} \\
\mathbf{M P a}\end{array}$ & $\begin{array}{c}\sigma \\
\mathbf{m}^{2} / \mathbf{g}\end{array}$ \\
\hline Cotta / Cottaer & 7.0 & 22.50 & 2080 & 21.6 & 52.3 & 5.26 \\
\hline Maulbronn / Maulbronner & 6.8 & 29.17 & 2120 & 21.7 & 65.0 & 6.52 \\
\hline Reinhardtsdorf / Reinhardtsdorfer & 8.8 & 182.67 & 1950 & 26.1 & 38.4 & 1.93 \\
\hline Posta / Postaer & 5.6 & 167.33 & 2110 & 20.3 & 79.7 & 0.06 \\
\hline Pliezhaus / Pliezhauser & 5.6 & 48.17 & 2090 & 21.6 & 40.3 & 1.03 \\
\hline Pfinztal / Pfinztaler & 3.5 & 10.67 & 2360 & 10.6 & 121.6 & 3.06 \\
\hline Donzdorf / Donzdorfer & 9.9 & 22.83 & 1910 & 28.6 & 20.0 & 3.85 \\
\hline
\end{tabular}

$\mathrm{A}_{b}=$ absorción de agua bajo presión atmosférica (DIN EN 13755) / water absorption under atmospheric pressure (DIN EN 13755)

$C$ = coeficiente de absorción de agua por capilaridad (DIN EN 1925) / capillary water absorption coefficient (DIN EN 1925)

$\rho_{\mathrm{b}}=$ densidad aparente (DIN EN 1936) / bulk density (DIN EN 1936)

$\mathrm{p}=$ porosidad total (DIN EN 1936) / total porosity (DIN EN 1936)

$\mathrm{R}=$ resistencia a la compresión uniaxial (DIN EN 1926) / uniaxial compressive strength (DIN EN 1926)

ó = superficie específica (DIN ISO 9277) / specific surface area (DIN ISO 9277)

Número de muestras analizadas: 5-7 / Number of specimens tested: 5-7

\section{MÉTODOS}

Para el estudio se sometieron muestras de las distintas areniscas a una atmósfera con dióxido de azufre. Se registró periódicamente el cambio de masa de las muestras y al final de los experimentos, se determinó la concentración de azufre de éstas. En cuatro de las siete muestras, se realizaron experimentos adicionales en que se analizaba sólo la matriz arcillosa.

Las muestras constaban de discos de $45 \mathrm{~mm}$ de diámetro y $3 \mathrm{~mm}$ de grosor cortados perpendicularmente al plano de estratificación. No se recubrieron las probetas a fin de que todas sus caras estuvieran expuestas a la atmósfera. Se colocaron las muestras sobre una rejilla dentro de una cámara de plexiglás por la que fluía aire con $\mathrm{SO}_{2}$. La concentración de $\mathrm{SO}_{2}$ era de $1,9 \mathrm{ppm}$ $\left(=5000 \mu \mathrm{g} / \mathrm{m}^{3}\right)$, la temperatura se mantuvo constante a $22{ }^{\circ} \mathrm{C}$ y se ajustó el flujo del gas a un valor constante de $12 \mathrm{l} / \mathrm{min}$. Había dos series de discos de cada uno de los siete tipos de arenisca. Uno se mantuvo a una humedad relativa del $65 \%$, mientras que el otro se humedeció semanalmente por ambos lados con 0,25 ml de agua bidestilada para que los poros tuvieran más agua para la reacción con la atmósfera, aparte del agua ya adsorbida.

\section{METHODS}

Specimens of each sandstone studied were exposed to a sulphur dioxide atmosphere. Changes in sample mass were recorded periodically and the sulphur concentration in the samples was determined upon termination of the trial. Further trials were conducted on the clayey matrix of only four of the seven sandstones.

The specimens were 45-mm diameter, 3-mm thick discs, cut perpendicularly to the bedding plane. The specimens were not coated; i.e., all sides were exposed to the air. Samples were placed on a rack inside a plexiglas chamber with an $\mathrm{SO}_{2}$ atmosphere, where the $\mathrm{SO}_{2}$ concentration was $1.9 \mathrm{ppm}\left(=5000 \mu \mathrm{g} / \mathrm{m}^{3}\right)$ and the temperature a constant $22{ }^{\circ} \mathrm{C}$. The gas flow was likewise constant, at $12 \mathrm{l} / \mathrm{min}$. Two sets of discs were prepared for each of the seven sandstone types. One set was stabilized at a relative humidity of $65 \%$, while the other was moistened weekly on both sides with $0.25 \mathrm{ml}$ of bidistilled water to ensure that the pores were kept moist for reaction with the atmosphere, irrespective of any pre-existing adsorbed water. 
El tiempo total de exposición fue de 96 días. El cambio de masa se registraba periódicamente (normalmente cada semana) sacando las muestras de la cámara de exposición y pesándolas. Una vez pesadas, se humedecían las muestras de la segunda serie y volvían a introducirse todas las muestras en la cámara. La humedad relativa era normalmente del $65 \%$, pero después de volver a introducir las muestras humedecidas, la HR aumentaba hasta el $75 \%$ durante uno o dos días. Después del experimento, las muestras se secaron a $70^{\circ} \mathrm{C}$ hasta alcanzar el balance de materia, se pesaron por última vez, se molieron hasta conseguir un polvo fino y se analizó su contenido de azufre con un analizador de carbono y azufre. El secado a $70{ }^{\circ} \mathrm{C}$ garantizaba que no se analizase el azufre que simplemente hubiera sido adsorbido por la superficie interna del sustrato pétreo.

A partir de la concentración final de azufre en las muestras, su tamaño y el tiempo de exposición se determinaron el flujo de deposición $(F)$ en $\mu \mathrm{g} /\left(\mathrm{cm}^{2} \mathrm{~d}\right)$ y la velocidad de deposición (v) en cm/s. Según (14), el flujo y la velocidad de deposición se definen como [1]:

$$
\mathrm{F}=\mathrm{m}_{\mathrm{SO}_{2}} /(\mathrm{A} \cdot \mathrm{t})
$$

donde $\mathrm{m}_{\mathrm{SO}_{2}}=$ materia absoluta de $\mathrm{SO}_{2}$ depositada en una muestra; $A=$ superficie de la muestra; $t=$ tiempo de exposición

$\mathrm{C}_{\mathrm{SO}_{2}}=$ concentración de $\mathrm{SO}_{2}$ en el aire

$$
\mathrm{v}=\mathrm{F} / \mathrm{C}
$$

Se extrajo la fracción arcilla de cuatro de las areniscas (Cotta, Maulbronn, Reinhardtsdorf y Pfinztal) y se expuso por separado a una atmósfera con la misma concentración de $\mathrm{SO}_{2}$ con el fin de investigar con mayor detalle la influencia de las fases de óxido o hidróxido de hierro en la matriz de cementante. La fracción arcilla se extrajo triturando las muestras de arenisca y dispersándolas en agua mediante técnicas de ultrasonidos. Posteriormente se eliminó la fracción gruesa mediante cribado. Se extrajo la fracción arcilla $\leq 2 \mu \mathrm{m}$ de la dispersión acuosa mediante centrifugación a una velocidad y con un tiempo determinados (véase (19) para más información), y se secó a $70^{\circ} \mathrm{C}$ hasta alcanzar el balance de materia. Aparte de eso, se trató de manera especial una muestra de la fracción arcilla de las dos areniscas con mayor contenido de hierro (Maulbronn y Pfinztal, véase Tabla 2). Se extrajeron de esos dos tipos de arenisca fases de óxido de hierro tratando la fracción arcilla con hidrosulfito sódico, según (19). A continuación se colocaron todas las muestras en polvo del aglomerado en la cámara con $\mathrm{SO}_{2}$. Las condiciones de trabajo eran las mismas que se han descrito anteriormente $\left(\mathrm{T}=22{ }^{\circ} \mathrm{C}, \mathrm{HR}=65 \%, \mathrm{C}_{\mathrm{SO}_{2}}=\right.$ $1,9 \mathrm{ppm})$. Esta segunda parte del experimento tuvo una duración de 17 días. Durante el mismo se registró el incremento de masa de las muestras. Después de haberse sometido al tratamiento, las muestras se secaron a $70{ }^{\circ} \mathrm{C}$ hasta alcanzar el balance de materia, se pesaron por última vez y se analizó su contenido de azufre con un analizador de carbono y azufre.
The total duration of exposure was 96 days. Changes in mass were recorded periodically (usually weekly) by weighing the specimens after temporary removal from the chamber. The second series of specimens were moistened prior to return to the chamber after weighing. Relative humidity was normally $65 \%$, but after reinsertion of the moistened samples it rose to $75 \%$ for one to two days. After the experiment the specimens were dried at $70{ }^{\circ} \mathrm{C}$ until the mass stabilized, weighed for the last time and then ground to a fine powder to analyze the sulphur content in a carbon sulphur analyzer (CSA). Drying at $70^{\circ} \mathrm{C}$ ensured that sulphur merely adsorbed on the inner surface of the stone substrate would not be analyzed.

Deposition flux ( $F$ ) in $\mu \mathrm{g} /\left(\mathrm{cm}^{2} d\right)$ and deposition velocity ( $v$ ) in $\mathrm{cm} / \mathrm{s}$ were determined from the sample size, final sulphur concentration and exposure time. According to (14) deposition flux and velocity are defined as [1]:

where $\mathrm{m}_{\mathrm{SO}_{2}}=$ absolute mass of $\mathrm{SO}_{2}$ deposited on a specimen; $A=$ sample area; $t=$ exposure time

\section{[2]}

$$
\mathrm{C}_{\mathrm{SO}_{2}}=\mathrm{SO}_{2} \text { concentration in the air }
$$

The clay fractions of four sandstones (Cottaer, Maulbronner, Reinhardsdorfer, Pfinztaler) were extracted and separately exposed to an atmosphere with the same $\mathrm{SO}_{2}$ concentration to analyze the effect of iron oxide/hydroxide phases on the binder matrix in greater detail. The clay fraction was extracted by crushing the sandstone specimens and dispersing the product in water with an ultrasonic disperser. The coarse fraction was then removed by sieving. The clay fraction $(\leq 2 \mu \mathrm{m})$ was centrifuged out of the aqueous dispersion at a predetermined revolution speed and time (see (19) for details) and then dried at $70^{\circ} \mathrm{C}$ until the mass stabilized. In addition, one batch each of the clay fractions of the two sandstones with the highest iron content (Maulbronner, Pfinztaler, see Table 2) was treated with sodium dithionite as described in (19) to remove the iron oxides. All the powdery binder fraction samples were then exposed to $\mathrm{SO}_{2}$ in the chamber under the conditions described above ( $T=22{ }^{\circ} \mathrm{C}, \mathrm{RH}=65 \%, \mathrm{C}_{\mathrm{SO}_{2}}=1,9 \mathrm{ppm}$ ) for 17 days. Changes in specimen mass were recorded during the trial. After exposure, the samples were dried at $70{ }^{\circ} \mathrm{C}$ until the mass stabilized, weighed for the last time and analyzed for sulphur content with CSA. 


\section{RESULTADOS EXPERIMENTALES}

\section{Discos de arenisca}

Los distintos tipos de arenisca mostraron un comportamiento muy variado durante el experimento. En la Figura 1 puede apreciarse el incremento de masa de los discos. En la serie de muestras secas se registró un aumento de masa en todos los casos a los pocos días. Las únicas areniscas que presentaron un aumento de masa de forma continuada después de los 30 días eran las de Maulbronn (M) y Pfinztal (Pf). En la primera el aumento de masa era superior a los $2 \mathrm{mg} / \mathrm{cm}^{2}$ a los 96 días y en la arenisca de Pfinztal de $0,86 \mathrm{mg} / \mathrm{cm}^{2}$. El incremento de masa registrado en los demás tipos de arenisca era inferior a los 0,5 $\mathrm{mg} / \mathrm{cm}^{2}$. Curiosamente, la arenisca de Pliezhaus (PI), pese a su contenido de cemento carbonatado, no presentó un aumento de masa especialmente importante al final de la prueba. Apenas se registraron cambios de masa en las areniscas de Posta (Po) y Reinhardtsdorf (R).

\section{RESULTS}

\section{Sandstone discs}

Sandstone behaviour varied substantially during the experiment, depending on the type of stone. Figure 1 shows the changes in disc mass. Gains were recorded for all the dry series specimens after only a few days. These gains were ongoing after 30 days in only two of the stones, however: Maulbronner (M) and Pfinztaler (Pf). The gain in mass in the former was upward of $2 \mathrm{mg} / \mathrm{cm}^{2}$ after the 96th day of exposure. The mass gain in Pfinztaler sandstone was $0.86 \mathrm{mg} / \mathrm{cm}^{2}$, while all the other sandstones exhibited gains of under $0.5 \mathrm{mg} / \mathrm{cm}^{2}$. The mass gain in the Pliezhauser stone (PI), which contained carbonate cement, was not particularly high after 96 days, while practically no gain at all was recorded for the Postaer $(P o)$ or Reinhardsdorfer $(R)$ stones.

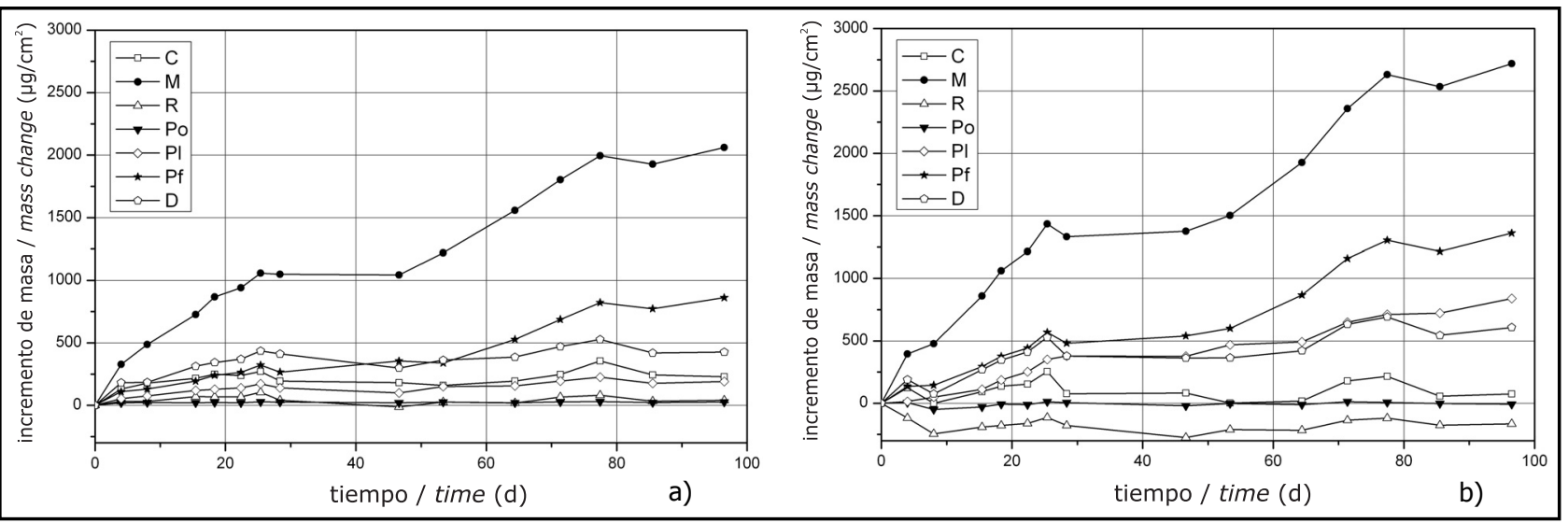

Figura 1. Incremento de masa de los discos de arenisca secos (a) y humedecidos (b) durante los ensayos, en función del tiempo. Consulte las abreviaturas en la tabla 1.

Figure 1. Time vs. change in mass of dry (a) and wet (b) sandstone discs during exposure. (See Table 1 for meaning of labels).

Más variación presentaron las curvas correspondientes a la serie de muestras que se humedecían periódicamente. De entre los discos humedecidos de arenisca, el aumento de masa mayor, con diferencia, se observó en los de Maulbronn, con $2,7 \mathrm{mg} / \mathrm{cm}^{2}$ al final del ensayo, lo que supone un $30 \%$ más que el aumento experimentado por los discos secos. La arenisca de Pfinztal registró el segundo mayor aumento de masa $\left(1,36 \mathrm{mg} / \mathrm{cm}^{2}\right)$, seguida de las probetas de Donzdorf y Pliezhaus. La reacción de ésta última en la atmósfera de $\mathrm{SO}_{2}$ fue mucho más intensa en estado húmedo que en estado seco. Al igual que en los discos secos, en los discos humedecidos de arenisca de Cotta (C) se observó sólo un ligero aumento de masa, que se estabilizó a los pocos días. No hubo incrementó
The mass change curves for the series of specimens that were periodically moistened were more diversified. By far the highest gain in mass was observed for wet Maulbronner sandstone, with $2.7 \mathrm{mg} / \mathrm{cm}^{2}$ by the end of the trial, i.e., 30\% higher than in the dry disc. Pfinztaler sandstone, with $1.36 \mathrm{mg} / \mathrm{cm}^{2}$, exhibited the next highest mass increase, followed by Donzdorfer and Pliezhauser. The latter stone reacted much more intensely with the $\mathrm{SO}_{2}$ in the atmosphere in the wet than in the dry state. As in the dry discs, the moistened Cottaer sandstone (C) specimens exhibited only a slight mass gain, which flattened after very few days. No change in mass was observed in Postaer sandstone. In the Reinhardsdorfer specimens, in turn, the mass decrease due to the detachment 
alguno en la masa de la arenisca de Posta. Las muestras de arenisca de Reinhardtsdorf incluso perdieron algo de masa debido a la exfoliación de algunos granos al humedecer las muestras, aunque a nivel global la diferencia fue imperceptible.

En la Figura 2 se muestra el contenido de azufre (=concentración de $\mathrm{SO}_{3}$ ) de las muestras tras los 96 días de ensayo. Los niveles de $\mathrm{SO}_{3}$ más altos fueron los de la arenisca de Maulbronn, en la que se alcanzó un $0,45 \%$ y un $0,62 \%$ de la masa (en las muestras secas y húmedas, respectivamente). La evolución de la concentración de $\mathrm{SO}_{3}$ en las distintas areniscas es básicamente igual a la del incremento de la masa (Figura 1) con una única excepción: los discos humedecidos de arenisca de Pliezhaus tenían mayor concentración de $\mathrm{SO}_{3}(0,43 \%$ de la masa) que la arenisca de Pfinztal (0,29\% de la masa). Esto se debió a que el aumento de masa durante los of a few grains during moistening. This decrease was barely perceptible, however.

The sulphur content (expressed as $\mathrm{SO}_{3}$ concentration) in the specimens after 96 days is shown in Figure 2. The highest $\mathrm{SO}_{3}$ levels were observed in Maulbronner sandstone, where concentration reached $0.45 \%$ (by mass, dry discs) and $0.62 \%$ (by mass, wet discs). The sulphate concentration curves followed essentially the same pattern as the change in mass curves (Figure 1), with one exception: the wet Pliezhauser sandstone discs had a higher $\mathrm{SO}_{3}$ concentration ( $0.43 \%$ by mass) than the Pfinztaler variety ( $0.29 \%$ by mass). The explanation for this difference is that the mass gain observed in the trials was not solely the net result of the replacement of $\mathrm{CO}_{2}$ by $\mathrm{SO}_{2}$ in

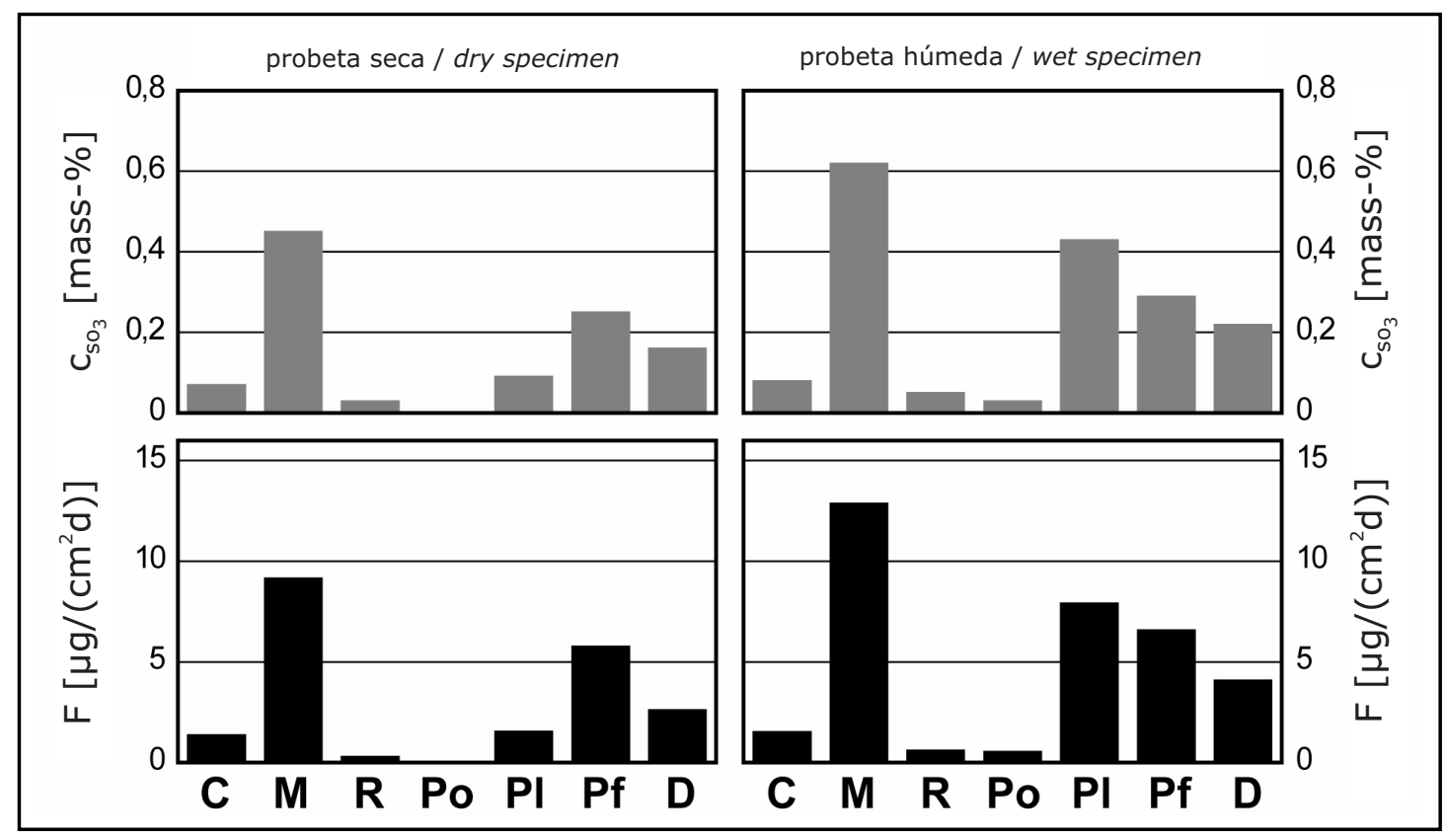

Figura 2. Concentración de azufre y flujo de deposición de $\mathrm{SO}_{2}$ de las areniscas estudiadas. Consulte las abreviaturas en la tabla 1.

Figure 2. Sulfur concentration and $\mathrm{SO}_{2}$ deposition flux for the sandstones studied. (See Table 1 for meaning of labels).

ensayos no estaba causado únicamente por el aumento de masa neto mediante pérdida de $\mathrm{CO}_{2}$ y fijación de $\mathrm{SO}_{2}$ en forma de sulfato, sino también por la adsorción de $\mathrm{SO}_{2}$ y agua a largo plazo. La Figura 2 muestra además los flujos de deposición, que fueron calculados a partir de la concentración final de $\mathrm{SO}_{2}$ en las muestras, la superficie de las muestras y el tiempo total del ensayo. Dado que la superficie de las muestras y el tiempo fueron idénticos para todas las muestras, el flujo es proporcional a la con- the form of sulphate, but also of the longterm adsorption of $\mathrm{SO}_{2}$ and water. Figure 2 also shows the deposition flux values estimated from the final $\mathrm{SO}_{2}$ concentration in the samples, the sample area and total time of exposure. Since the sample area and exposure time were identical in all the specimens, flux was proportional to the $\mathrm{SO}_{2}$ concentration. In all the dry specimens except Maulbronner and Pfinztaler, 
centración de $\mathrm{SO}_{2}$. Las muestras secas, con la excepción de las areniscas de Maulbronn y Pfinztal, tenían flujos muy por debajo de $5 \mu \mathrm{g} /\left(\mathrm{cm}^{2} \mathrm{~d}\right)$. Sin embargo, la humidificación periódica de las muestras aumentó el flujo en casi todas las areniscas.

Los resultados de los ensayos pusieron claramente de manifiesto que el aumento de humedad en las muestras mediante la humidificación periódica provocó también un aumento de la deposición y fijación de $\mathrm{SO}_{2}$ en los discos de arenisca, lo que concuerda con los datos registrados en otros trabajos $(23,24)$. También pudo observarse que dos de las areniscas, Cotta y Maulbronn, que tenían una cantidad parecida de matriz cementante y propiedades físicas muy parecidas (Tabla 3), presentaron comportamientos completamente diferentes en cuanto a la deposición de $\mathrm{SO}_{2}$. Dado que esto sólo puede justificarse por las diferencias en la composición del aglomerado, se decidió realizar los ensayos que se describen a continuación, en los que sólo se expusieron a una atmósfera con $\mathrm{SO}_{2}$ los cementos de algunas de las areniscas.

\section{Aglomerado}

Se seleccionaron los aglomerados de cuatro tipos de arenisca por su composición específica. La masa de dos de las areniscas (Maulbronn y Pfinztal) contenía hematites $\left(\mathrm{Fe}_{2} \mathrm{O}_{3}\right)$. La arenisca de Reinhardtsdorf contenía como principal fase férrica el hidróxido lepidocrocita $(\mathrm{FeO}(\mathrm{OH}))$. En la arenisca de Cotta, la mayor parte del hierro del aglomerado estaba fijada como $\mathrm{Fe}^{++}$en glauconita y otros minerales arcillosos, pero no se detectaron óxido ni hidróxidos de hierro.

En la Figura 3 pueden verse los resultados del ensayo de los aglomerados. Los de Maulbronn (M) y Pfinztal (Pf) presentaron el mayor aumento de masa durante el ensayo, mostrando una tendencia positiva. El incremento de masa de los otros dos tipos de arenisca (Cotta, C; Reinhardtsdorf, R) se estabilizó en alrededor del $1 \%$ tras sólo 3 días de ensayo. Sin embargo, se observó que en la muestra del aglomerado de las areniscas de Maulbronn (M-Fe) y de Pfinztal (Pf-Fe) que carecía de hematites, los valores de la velocidad de deposición de dióxido de azufre oscilaban dentro de exactamente el mismo intervalo que los del material de Cotta y Reinhardtsdorf. La concentración de azufre al final del ensayo siguió un patrón similar. Las areniscas de Maulbronn y Pfinztal presentaban las concentraciones más elevadas (entre el $1,7 \%$ y el $2 \%$ de la masa), mientras que las muestras sin hematites y las otras dos areniscas tenían el mismo contenido de $\mathrm{SO}_{3}$ (aproximadamente el $0,5 \%$ de la masa).

Esto indicaba claramente la influencia del óxido de hierro en forma de hematites en la deposición de $\mathrm{SO}_{2}$ en las areniscas. Sin embargo, el hidróxido de hierro (lepidocro- flux was well below $5 \mu \mathrm{g} /\left(\mathrm{cm}^{2} d\right)$. Routine moistening increased flux in nearly all the sandstones, however.

The trials clearly showed that the increased moisture deriving from periodic wetting also prompted a rise in $\mathrm{SO}_{2}$ deposition on and fixation in sandstone discs. These results concur with findings reported by other authors $(23,24)$. Another interesting observation was that $\mathrm{SO}_{2}$ deposition was completely different in two of the sandstones, Cottaer and Maulbronner, despite their similarities in terms of the proportion of binder matrix and physical properties (Table 3). The only possible explanation would be the existence of differences in their binder composition. This was the chief reason for conducting the following experiments, in which the binder fractions of selected sandstones were exposed to an $\mathrm{SO}_{2}$ atmosphere.

\section{Binder fraction}

The binder fractions of the four sandstones were chosen for their specific composition. Two, Maulbronner and Pfinztaler, contained hematite $\left(\mathrm{Fe}_{2} \mathrm{O}_{3}\right)$. In the Reinhardsdorfer stone, in turn, iron was found predominantly in the form of ledipocrocite $(\mathrm{FeO}(\mathrm{OH}))$. Most of the iron in the Cottaer sandstone binder was fixed in the form of $\mathrm{Fe}++$ in glauconite, and while other clay minerals were present, no iron oxide or hydroxides were detected.

The results of exposing the binder fractions to $\mathrm{SO}_{2}$ are shown in Figure 3. The greatest mass gains were found for the Maulbonner (M) and Pfinztaler (Pf) binder fractions, whose curves tended upward at the end of the trial. In the other two stones (Cottaer, $C$, and Reinhardsdorfer, $R$ ) mass gains flattened at around $1 \%$ after only three days. Nonetheless, sulphur dioxide deposition in the hematitefree batches of the Maulbronner ( $M-F e)$ and Pfinztaler (Pf-Fe) binder fractions fell within exactly the same range as in the Cottaer and Reinhardsdorfer fractions. Sulphur concentration at the end of the trial followed an analogous pattern. The highest concentrations were observed for Maulbronner and Pfinztaler, with values of 1.7 to $2 \%$ by mass, while the hematite-free batches of these two stones and the other two varieties had concentrations of around $0.5 \%$ by mass.

This provided clear proof of the effect of iron oxide in the form of hematite on $\mathrm{SO}_{2}$ deposition in these sandstones. The iron hydroxide (lepidocrocite) in the binder fraction 
cita) del aglomerado de la arenisca de Reinhardtsdorf parecía no producir el mismo efecto que las hematites de los otros dos materiales, ya que la deposición de $\mathrm{SO}_{2}$ en la primera era mucho menor. Los resultados indican asimismo que los minerales arcillosos no parecen incidir de manera apreciable en la deposición inicial de dióxido de azufre. of Reinhardsdorfer sandstone seemed not to have the same effect as the hematite in the other two materials, for $\mathrm{SO}_{2}$ deposition was much lower in the former. These results also appear to rule out any major role of clay minerals in initial sulphur dioxide deposition.

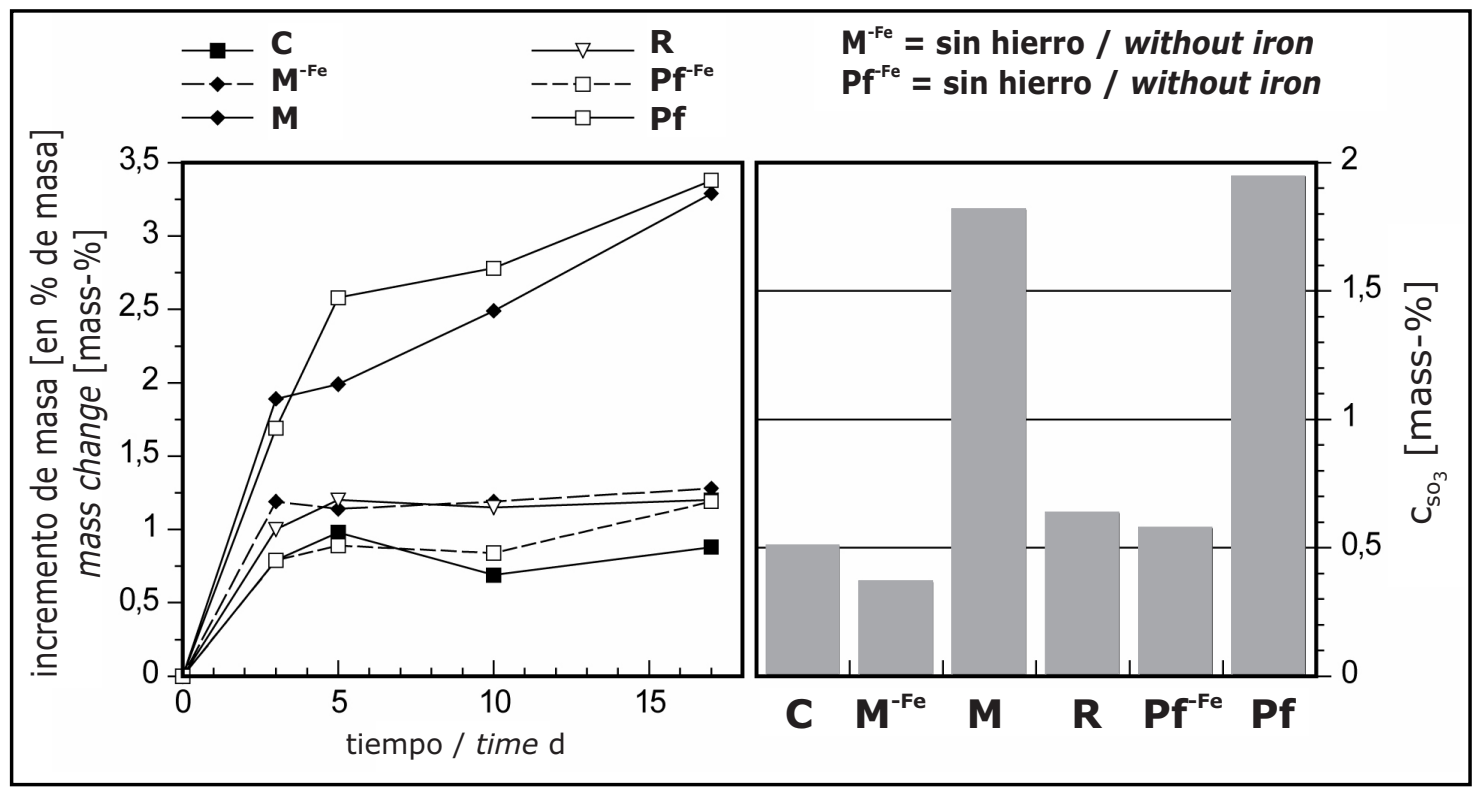

Figura 3. Incremento de masa en función del tiempo y de la concentración de azufre del aglomerado de cuatro areniscas.

Figure 3. Change in mass vs. time and sulphur concentration in the binder fractions of four sand-stones.

\section{DISCUSIÓN Y CONCLUSIONES}

Los resultados han demostrado que los siete tipos de arenisca reaccionaron de forma distinta en lo que a la deposición del dióxido de azufre se refiere. Un parámetro fundamental parece ser el contenido de óxidos de hierro en el aglomerado. Otro factor importante es la superficie específica, que posiblemente mejore la adsorción de $\mathrm{SO}_{2}$ en las areniscas, fomentando de este modo la fijación temprana de $\mathrm{SO}_{2}$ en el aglomerado. En la Tabla 3 se enumeran las superficies específicas de los distintos materiales. Como puede verse, los valores varían entre los 0,06 $\mathrm{m}^{2} / \mathrm{g}$ (Posta) y los 6,52 m²/g (Maulbronn). Con objeto de evaluar la eventual correlación entre la superficie específica y la deposición de $\mathrm{SO}_{2}$ se ha representado la superficie en función del flujo de deposición en el grafico de la Figura 4, en la que también se traza el contenido total de hierro de las areniscas en función de F. Queda patente que no existe ninguna correlación entre $F$ y la superficie específica; sin embargo, la concentración total de hierro y $\mathrm{F}$ muestran una cierta correlación tanto para las muestras secas como las humedecidas (indicadas con la línea

\section{DISCUSSION AND CONCLUSIONS}

The trials showed that sulphur dioxide deposition differed in the seven sandstones studied. One crucial parameter seemed to be the iron content in the form of oxides in the binder fraction. The specific surface area also proved to be an important variable for its role in the adsorption of $\mathrm{SO}_{2}$ and its contribution to the early fixation of the compound in the binder matrix. The specific surface area of the various materials given in Table 3 ranged from 0.06 $\mathrm{m}^{2} / \mathrm{g}$ (Postaer) to $6.52 \mathrm{~m}^{2} / \mathrm{g}$ (Maulbronner). Surface area was plotted against flux (Figure 4) to determine the possible correlation between specific surface area and $\mathrm{SO}_{2}$ deposition. Figure 4 also relates the total iron content to $F$. These results clearly indicate that there was no correlation between $F$ and specific surface area. Total iron concentration and $F$, however, were fairly closely correlated in both the dry and wet samples (dotted line). Behaviour was observed to be different in the carbonatecontaining Pliezhauser sandstone only, due to the higher 
de puntos). Sólo la arenisca de Pliezhaus, que contiene carbonato, presenta un comportamiento distinto debido a la mayor velocidad de reacción entre el $\mathrm{SO}_{2}$ y el cemento carbonatado, sobre todo en las muestras humedecidas. reaction rate between $\mathrm{SO}_{2}$ and the carbonate cement, in particular in wet specimens.

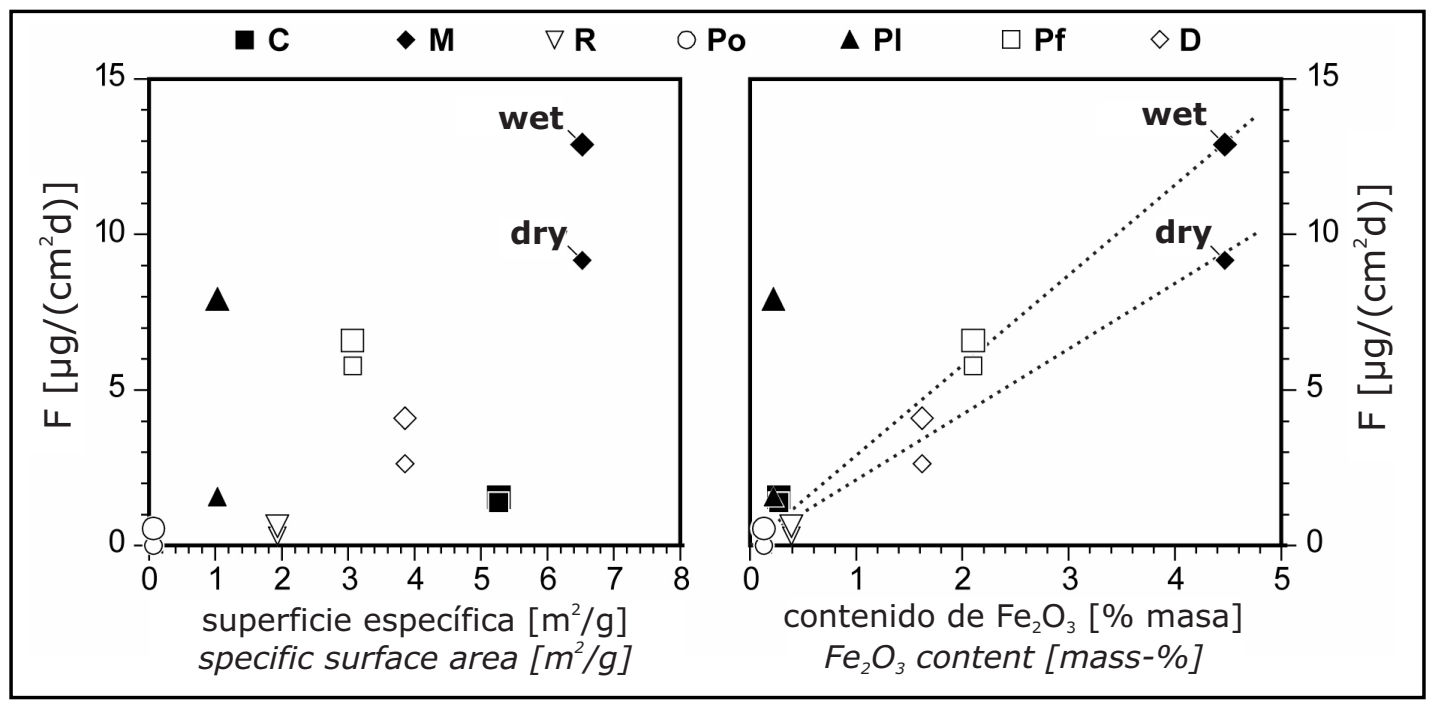

Figura 4. Superficie específica y contenido de hierro de las areniscas en función del flujo de deposición. Consulte las abreviaturas en la Tabla 1.

Figure 4. Sandstone specific surface area and iron content vs. deposition flux. (See Table 1 for meaning of labels).

Debe tenerse en cuenta que el contenido total de hierro no es necesariamente el mismo que el contenido de óxidos o hidróxidos de hierro en las muestras; pero en el caso de las areniscas con mayor contenido de hierro la mayor parte del hierro total se encontraba en forma de óxidos o hidróxidos, lo que explica el color marrón rojizo de las areniscas de Maulbronn y Pfinztal (debido a las hematites) y el color marrón amarillento de la arenisca de Donzdorf (debido a la goetita).

Haneef y otros $(15,16)$ ya sospechaban que las impurezas de la piedra en forma de óxidos podrían actuar como catalizador para la oxidación de $\mathrm{SO}_{2}$ a $\mathrm{SO}_{3}$ según esta reacción [3]:

$$
\mathrm{SO}_{2}(\mathrm{~g}) \stackrel{\text { disolución (rápida) / dissolution (fast) }}{\longrightarrow} \mathrm{SO}_{2}(\mathrm{aq}) \stackrel{\mathrm{O}_{2} / \text { catalizador (rápida) / catalyst (fast) }}{\longrightarrow} \mathrm{SO}_{3}(\mathrm{aq}) \longrightarrow \mathrm{H}_{2} \mathrm{SO}_{4}
$$

Los resultados presentados demuestran que esta suposición es válida y que las hematites pueden acelerar la reacción anterior. Otros óxidos, como el de manganeso, también podrían actuar como catalizador, pero en todas las areniscas analizadas, la concentración de MnO era inferior al 0,02\% de la masa. Las partículas transportadas por el aire, tales como las cenizas volantes, también pueden provocar un aumento de las velocidades de deposición
It should be stressed that the total iron content is not necessarily the same as the iron oxides or hydroxide content in the specimens. In sandstone with a high iron content, however, most of the total iron is bonded to oxides/hydroxides, as attested by the hematite-induced reddish brown hue of the Maulbronner and Pfinztaler stones and the goethite-mediated yellowish brown colour of Donzdorfer sandstone.

Haneef et al. $(15,16)$ suggested that oxide impurities in the stone may catalyze the oxidation of $\mathrm{SO}_{2}$ to $\mathrm{SO}_{3}$, as follows [3]:
The results presented here show that their assumption is valid and hematite greatly accelerates the above reaction. Other oxides, such as manganese, may also act as catalysts, but in all the sandstones studied the MnO concentration was under $0.02 \%$ by mass. Airborne particles such as fly ash may also raise $\mathrm{SO}_{2}$ deposition rates (20) in sandstone. Soot particles seem to cause an effect contrary to the interpretation given by Ausset et al. (20), as 
(20) en la arenisca. Sin embargo, las partículas de hollín parecen tener el efecto contrario, tal y como interpretaron Ausset y otros (20), al formar una capa protectora sobre la superficie de la piedra. Los resultados del presente estudio ponen de manifiesto que la velocidad de deposición de $\mathrm{SO}_{2}$ en las areniscas con hematites era de casi una orden de magnitud superior a la de las areniscas sin hematites. Los hidróxidos de hierro, sin embargo, parecen tener un efecto catalizador menor sobre la deposición de $\mathrm{SO}_{2}$. Una posible reacción en masa en la que las hematites actuaran como catalizador activo podría ser ésta [4]: they form a protective layer on the stone surface. According to the findings of the present study, $\mathrm{SO}_{2}$ deposition rates were nearly a magnitude higher in hematitebearing than in hematite-free sandstones. The catalytic effect of iron hydroxides on $\mathrm{SO}_{2}$ deposition appears to be smaller, however. One possible formulation of the reaction in which hematite intervenes as a catalyst would be as follows [4]:

$$
\begin{aligned}
& \mathrm{SO}_{2}+\mathrm{Fe}_{2} \mathrm{O}_{3}+3 \mathrm{H}_{2} \mathrm{O} \rightarrow \mathrm{SO}_{4}^{2-}+2 \mathrm{FeO}+2 \mathrm{H}_{3} \mathrm{O}^{+} \\
& 2 \mathrm{FeO}+1 / 2 \mathrm{O}_{2} \rightarrow \mathrm{Fe}_{2} \mathrm{O}_{3} \\
& \mathrm{SO}_{2}+1 / 2 \mathrm{O}_{2}+3 \mathrm{H}_{2} \mathrm{O} \rightarrow \mathrm{SO}_{4}^{2-}+2 \mathrm{H}_{3} \mathrm{O}^{+}
\end{aligned}
$$

No se investigó si la reacción anterior transformaba el $\mathrm{SO}_{2}$ directamente en $\mathrm{SO}_{4}{ }^{2-}$ o mediante la conversión a $\mathrm{SO}_{3}$ en solución acuosa.

Sin embargo, los resultados presentados aquí no concuerdan con los de Böke y otros (17), que no detectaron efecto catalizador alguno por parte de los óxidos de hierro cuando éstos se mezclaban con polvo de mármol. Dicho resultado podría deberse a la elevada concentración de $\mathrm{SO}_{2}$ empleada (3500 ppm), que era casi 1500 veces superior que la concentración de $\mathrm{SO}_{2}$ usada en el presente estudio. Una concentración tan elevada puede ocultar fácilmente toda función catalizadora de los óxidos por la deposición de $\mathrm{SO}_{2}$ en forma de sulfitos de calcio en vez de sulfatos. Por otra parte, Urone y otros (21) observaron un fuerte aumento de la velocidad de reacción en sus trabajos con óxidos puros de distintos metales (incluido el hierro) y una atmósfera con $\mathrm{SO}_{2}$ (en una concentración de 19 ppm).

La fijación de $\mathrm{SO}_{2}$ se conoce bastante bien en las piedras calcáreas. En el caso de la arenisca de Pliezhaus, la sulfatación del cemento de dolomía y calcita es el mecanismo predominante para la fijación de $\mathrm{SO}_{2}$ en forma de yeso. En este proceso debe tenerse en cuenta el comportamiento distinto de la dolomía y la calcita, como se describe en (12). Sin embargo, los mecanismos de fijación del dióxido de azufre en la arenisca no calcárea son menos conocidos. Una de las incógnitas es la procedencia de los cationes una vez el dióxido de azufre se ha oxidado a trióxido de azufre. En las areniscas arcillosas, los minerales arcillosos podrían desempeñar una función importante. Por una parte, las arcillas expansivas pueden intercambiar iones, como ocurre con la esmectita o con arcillas mixtas estratificadas con contenido de esmectita. En el caso de la reacción anterior, los cationes intercambiables (p. ej. $\mathrm{Ca}^{2+}, \mathrm{Mg}^{2+}, \mathrm{Na}^{+}, \mathrm{K}^{+}$) intercalados entre las capas arcillosas pueden intercambiarse por $\mathrm{H}_{3} \mathrm{O}^{+}$y quedar por tanto disponibles para la precipitación de sales de sulfato. Otro mecanismo podría ser la hidrólisis de silica-
The question of whether the above reaction oxidized $\mathrm{SO}_{2}$ directly to $\mathrm{SO}_{4}^{2-}$ or to $\mathrm{SO}_{3}$ in an aqueous solution was not addressed here.

In any event, these results do not concur with the findings reported by Böke et al. (17), who observed no actual catalytic action attributable to iron oxides when mixed with marble powder. This may have been due, however, to the high (3500 ppm) $\mathrm{SO}_{2}$ concentration used, nearly 1500 times higher than in the present study. At such a concentration, the deposition of $\mathrm{SO}_{2}$ in the form of calcium sulphites instead of sulphates could easily mask oxide-mediated catalysis. Moreover, in experiments with the pure oxides of several metals (including iron) and an atmosphere with $\mathrm{SO}_{2}$ (at a concentration of 19 ppm) Urone et al. (21) observed a substantial increase in the reaction rate.

The fixation of $\mathrm{SO}_{2}$ is fairly well understood in calcareous stone. In Pliezhauser sandstone, dolostone/calcite cement sulphation is the dominant mechanism for the fixation of $\mathrm{SO}_{2}$ in the form of gypsum The differences in dolomite and calcite behaviour as described in (12) are instrumental to this process. The mechanisms involved in sulphur dioxide fixation in non-calcareous sandstone are less well understood, however. One question outstanding solution is the origin of the cations when sulphur dioxide is oxidized to trioxide. The clay minerals in clayey sandstones may play a significant role in this regard. On the one hand, swelling clays such as smectite or mixed layered clays with a smectite component have ion exchange capacities. In the above reaction, exchangeable cations such as $\mathrm{Ca}^{2+}, \mathrm{Mg}^{2+}, \mathrm{Na}^{+}$or $\mathrm{K}^{+}$interspersed in the clay interlayer may be exchanged for $\mathrm{H}_{3} \mathrm{O}^{+}$, whereafter they precipitate as sulphate salts. Another mechanism might be the hydrolysis of aluminium silicates such as feldspars, clay minerals or micas in acid solutions as described in (22). Since the oxidation of $\mathrm{SO}_{2}$ to $\mathrm{SO}_{3}$ in an aqueous 
tos de aluminio, como feldespatos, minerales arcillosos o micas en una solución ácida, como se describe en (22). Puesto que la oxidación de $\mathrm{SO}_{2}$ a $\mathrm{SO}_{3}$ en una disolución acuosa provoca un aumento de la acidez, dichos aluminosilicatos podrían liberar álcalis y cationes alcalinotérreos que se disolverían, precipitando posteriormente en forma de sulfatos dentro de los poros o en la superficie de las areniscas. Por tanto, las areniscas no calcáreas más sensibles a la deposición de $\mathrm{SO}_{2}$ serían aquéllas con hematites y arcilla esmectítica en sus aglomerados, como en el caso de la arenisca de Maulbronn. solution entails a rise in acidity, alkali and alkali earth cations released from the aluminium silicates may dissolve and subsequently precipitate in the form of sulphates in the pore structure or on the surface of sandstone. The non-calcareous sandstones most sensitive to $\mathrm{SO}_{2}$ deposition would consequently be stones containing hematite and smectic clay in the binder fraction, such as in the case of Maulbronner sandstone.

\section{BIBLIOGRAFÍA / BIBLIOGRAPHY}

(1) Pettijohn, F. J., Potter, P. E., Siever, R.: "Sand and Sandstone", $2^{\mathrm{a}}$ ed., Springer Verlag, Nueva York, (1987), p. 553.

(2) Mausfeld, S. A., Grassegger, G.: "Abbauprozesse an Feldspäten und Tonmineralen unter den Bedingungen der Bauwerksverwitterung".

Zeitschrift der Deutschen Gesellschaft für Geowissenschaften, vol. 143, (1992), p. 23-39.

(3) Grassegger, G.: "Mineralogische Prozesse bei der Bausteinverwitterung", En: Wittenburg, C., Behlen, A., Steiger, M., Dannecker, W. (ed.): Denkmalpflege und Naturwissenschaft, Natursteinkonservierung II, Snethlage, R.: Verbundforschungsprojekt Steinzerfall und Steinkonservierung, Fraunhofer IRB Verlag, (1998), p. 119-136.

(4) Grassegger, G.: "Decay mechanisms of natural building stones on monuments - A review of the latest theories". En: Große, C. (ed): Werkstoffe und Werkstoffprüfung im Bauwesen, Hamburgo, Libri BOD, (1999), p. 54-81.

(5) Müller, U., Althaus, E., Karotke, E.: "Einfluß des Porengefüges auf die Schadstoffverteilung in Werk-Sandsteinen". En: Wenzel, F. (ed.): Erhalten historisch bedeutsamer Bauwerke, Jahrbuch 1997/98, Berlín, Ernst \& Sohn, (2000), p. 107-120.

(6) Winkler, E. M.: "Important agents of weathering for building and monumental stone". Engineering Geology, vol. 1, no. 5, (1966), p. 381-400.

(7) Niesel, K.: "Zur Verwitterung von Baustoffen in schwefeldioxidhaltiger Atmosphäre - Literaturdiscussion". Fortschritte der Mineralalogie, vol. 57, (1979), p. 68-124.

(8) Webb, A. H., Bawden, R. J., Busby, A. K., Hopkins, J. N.: "Studies on the effects of air pollution on limestone degradation in Great Britain". Atmospheric Environment. Part B. Urban Atmosphere, vol. 26, no. 2, (1992), p. 165-181.

(9) Cobourn, W. G., Gauri, K. L., Tambe, S., Li, S., Saltik, E.: "Laboratory measurements of sulfur dioxide deposition velocity on marble and dolomite stone surfaces". Atmospheric Environment. Part B. Urban Atmosphere, vol. 27, no. 2, (1993), p. $193-201$.

(10) Dolske, D. A.: "Deposition of atmospheric pollutants to monuments, statues, and buildings". Science of the Total Environment, vol. 167, no 1-3, (1995), p. 15-31.

(11) Malaga-Starzec, K.: "Microscopic studies of the first steps in chemical weathering of marble, limestone and sandstone". En: Stamatakis, M., Georgali, B., Fragoulis, D., Toumbakari, E. E. (ed.): Proceedings of 8th Euroseminar Microscopy Applied to Buildings Materials, Atenas, (2001), p. 21-27.

(12) Malaga-Starzec, K., Panas, I., Lindqvist, O.: "Model study of initial adsorption of $\mathrm{SO}_{2}$ on calcite and dolomite". Applied Surface Science, vol. 222, no. 1-4, (2004), p. 82-88.

(13) Dannecker, W., Steiger, M.: "Der Einfluß von Luftschadstoffen auf die Verwitterung von Natursteinen". En: Snethlage, R. (ed): Jahresberichte Steinzerfall - Steinkonservierung 1989, Berlín, Verlag Ernst \& Sohn, (1991), p. 115-129.

(14) Wittenburg, C., Behlen, A., Steiger, M., Dannecker, W.: "Die Messung von Luftschadstoffen und deren Deposition auf verschiedene Sandsteine an historischen Bauwerken". En: Wittenburg, C., Behlen, A., Steiger, M. Dannecker, W. (ed.), Denkmalpflege und Naturwissenschaft, Natursteinkonsrevierung II, Snethlage, R.: Verbundforschungsprojekt Steinzerfall und Steinkonservierung, Fraunhofer IRB Verlag (1998), p. 27-60.

(15) Haneef, S. J., Johnson, J. B., Dickinson, C., Thompson, G. E., Wood, G. C.: "Effect of dry deposition of $\mathrm{NO}_{x}$ and $\mathrm{SO}_{2}$ gaseous pollutants on the degradation of calcareous building stones". Atmospheric Environment. Part A. General Topics, vol. 26, no. 16, (1992), p. $2963-2974$. (16) Haneef, S. J., Jones, M. S., Johnson, J. B., Thompson, G. E., . Wood, G. C.: "Effects of air pollution on historic buildings and monuments (1986-1990). Scientific basis for conservation: Laboratory chamber studies". European Cultural Heritage Newsletter on Research, vol. 7, no. 1-4, (1993), p. 2-10.

(17) Böke, H., Göktürk, H., Caner-Saltik, E. N.: "Effect of particulate matter on sulphation of calcareous stone in humid atmosphere containing sulphur dioxide". En: Riederer, J. (ed.): Proceedings of 8th International Congress on Deterioration and Conservation of Stone, Berlín, (1996), p. 407-414.

(18) Moore, D. M., Reynolds, R. C. J.: "X-ray diffraction and the identification and analysis of clay minerals", Oxford University Press, Oxford, United Kingdom, (1989), p. 332.

(19) Tributh, H., Lagaly, G.: "Identifizierung und Charakterisierung von Tonmineralen, Berichte der DTTG", vol. 1, Deutsche Ton- und Tonmineralgruppe e. V., Giessen (Alemania), (1991), p. 162. 
(20) Ausset, P., Crovisier, J. L., Del Monte, M., Furlan, V., Girardet, F., Hammecker, C., Jeannette, D., Lefevre, R. A.: "Experimental study of limestone and sandstone sulphation in polluted realistic conditions: The Lausanne Atmospheric Simulation Chamber (LASC)". Atmospheric Environment, vol. 30, no. 18, (1996), p. 3197-3207.

(21) Urone, P., Lutsep, H., Noyes, C. M., Parcher, J. F.: "Static studies of sulfur dioxide reactions in air". Environmental Science and Technology, vol. 2, no. 8, (1968), p. 611-618.

(22) Helgeson, H. C., Garrels, R. M.y MacKenzie, F. T.: "Evaluation of irreversible reactions in geochemical processes involving minerals and aqueous solutions - II. Applications". Geochimica et Cosmochimica Acta, vol. 33, no. 4 (1969), p. 455-481.

(23) Bai, Y., Thompson, G. E., Martínez Ramírez, S., Brueggerhoff, S.: "Mineralogical study of salt crusts formed on historic building stones". The Science of the Total Environment, vol. 302 (2003), p. 247-251.

(24) Martínez Ramírez, S., Puertas, F., Blanco Varela, M. T., Thomson, G. E.: "Effect of dry deposition of pollutants on the degradation of lime mortars with sepiolite". Cem. Concr. Res., vol. 28, nº 1 (1998), p. 125-133. 